\title{
Use of the Population Grouping Methodology of the Canadian Institute for Health Information to predict high-cost health system users in Ontario
}

\author{
Sharada Weir DPhil, Mitch Steffler MA, Yin Li PhD, Shaun Shaikh MA, James G. Wright MD MPH, \\ Jasmin Kantarevic PhD
}

Cite as: CMAJ 2020 August 10;192:E907-12. doi: 10.1503/cmaj.191297

\begin{abstract}
BACKGROUND: Prior research has consistently shown that the heaviest users account for a disproportionate share of health care costs. As such, predicting high-cost users may be a precondition for cost containment. We evaluated the ability of a new health risk predictive modelling tool, which was developed by the Canadian Institute for Health Information $(\mathrm{ClHI})$, to identify future highcost cases.
\end{abstract}

METHODS: We ran the CIHI model using administrative health care data for Ontario (fiscal years 2014/15 and 2015/16) to predict the risk, for each individual in the study population, of being a high-cost user 1 year in the future. We also estimated actual costs for the prediction period. We evaluated model performance for selected percentiles of cost based on the discrimination and calibration of the model.

RESULTS: A total of 11684427 individuals were included in the analysis. Overall, $10 \%$ of this population had annual costs exceeding $\$ 3050$ per person in fiscal year $2016 / 17$, accounting for $71.6 \%$ of total expenditures; $5 \%$ had costs above $\$ 6374$ (58.2\% of total expenditures); and 1\% exceeded \$22995 (30.5\% of total expenditures). Model performance increased with higher cost thresholds. The c-statistic was 0.78 (reasonable), 0.81 (strong) and 0.86 (very strong) at the $10 \%, 5 \%$ and $1 \%$ cost thresholds, respectively.

INTERPRETATION: The CIHI Population Grouping Methodology was designed to predict the average user of health care services, yet performed adequately for predicting high-cost users. Although we recommend the development of a purpose-designed tool to improve model performance, the existing $\mathrm{CIHI}$ Population Grouping Methodology may be used - as is or in concert with additional information - for many applications requiring prediction of future high-cost users.
A substantial literature across health systems shows that the highest users of services account for disproportionate shares of the public costs of health care. It has recently been reported that more than three-quarters of individual health care costs in Ontario were incurred by just $10 \%$ of the population. ${ }^{1}$ Similarly, an Ontario Ministry of Health and LongTerm Care (MOHLTC) analysis of inpatient and home care costs found that the top $5 \%$ of patients were responsible for $61 \%$ of spending in those domains. ${ }^{2}$ Consistent findings have been reported for Manitoba, Alberta and British Columbia. ${ }^{3-7}$

Some of the highest-cost cases may be explained by rare, unpredictable events, but others arise in the presence of multiple chronic conditions. Research from the United States has suggested that spending on chronic conditions accounts for the majority of health care expenditures. ${ }^{8}$ Predicting high-cost users may help us to understand and better manage public spending on health care.

Cognizant of this need, the Ontario MOHLTC developed a predictive model for high-cost users based on sociodemographic, utilization and clinical diagnostic characteristics. ${ }^{9}$ Although the model performed well, it relied on a coarse categorization of 20 diagnostic variables consisting of broadly defined chapters of the International Statistical Classification of Diseases and Related Health Problems, 10th Revision (ICD-10) and a small number of chronic conditions, which limited its utility for explaining predictions. Moreover, this model is not available for use outside the MOHLTC. As such, there is a need for a predictive model that can be applied more widely by 
researchers and other stakeholders with an interest in health policy and spending in Canada.

The Canadian Institute for Health Information $(\mathrm{CIHI})$ has recently released a new population-based case mix product, the Population Grouping Methodology, which uses diagnoses obtained from patient health care encounters in multiple settings to summarize the universe of diagnosis codes into a clinically meaningful set of 226 health conditions. The grouping and modelling methodologies are described in more detail in Appendix 1 (available at www.cmaj.ca/lookup/suppl/doi:10.1503/ cmaj.191297/-/DC1) and in previous reports. ${ }^{10,11}$ The CIHI grouping methodology was not designed to predict high-cost cases, and previous work has already shown that the model performs better for low- and moderate-cost users than for highest-cost users (i.e., those with annual costs exceeding $\$ 25000$ ). ${ }^{11}$ We evaluated the suitability of $\mathrm{CIHI}$ 's model for predicting future high-cost users in Ontario by examining the predicted costs for individuals who exceeded the top $10 \%, 5 \%$, and $1 \%$ thresholds of actual cost.

\section{Methods}

\section{Study design and setting}

The study design involved validation of $\mathrm{CIHI}$ 's cost-risk predictions for high-cost health system users in Ontario. The $\mathrm{CIHI}$ model was developed using a 3-province data set from 2010/11 through 2012/13. We used out-of-sample data representing the population of Ontario, one of the provinces that was included in model development. Using diagnoses recorded in the validation set over a look-back period of 2 fiscal years (2014/15 and 2015/16), we ran the CIHI model to predict high-cost individuals in the following year (2016/17) and then assessed model performance according to whether individuals who were predicted to have high costs actually had high costs, at selected cost thresholds (e.g., top 10\%). To avoid including individuals who had died or left the province without their change in eligibility being recorded in the Registered Persons Database, we limited the study population to Ontario residents under 106 years of age who had health care utilization during the look-back (baseline) period and were eligible for coverage under the Ontario Health Insurance Plan (OHIP) during the full study period.

\section{Data sources}

The Ontario MOHLTC provided administrative health care eligibility and encounters files. We combined data from the Registered Persons Database for eligible individuals with all available encounters from CIHI's Discharge Abstract Database, CIHI's National Ambulatory Care Reporting System and the OHIP physician billings database. Together, these sources provide information on almost all inpatient, outpatient and ambulatory encounters in Ontario.

\section{Identification of health conditions}

We processed diagnosis data using ClHI's Population Grouping Methodology software, which output a set of 226 health condition flags (Appendix 2, Table A2-1, available at www.cmaj.ca/lookup/ suppl/doi:10.1503/cmaj.191297/-/DC1), along with resource intensity weights from a model predicting cost in the next year using health conditions found in the look-back period. Resource intensity weights are relative risk scores created by dividing each individual's predicted cost by the average for the population, so that individuals (and subgroups) can be compared in terms of their expected utilization of health care resources.

To understand better the drivers of cost and risk, we examined the prevalence of chronic conditions among patients in the top risk groups and among those with the highest actual cost. To identify chronic conditions, we included the 85 of the 226 health conditions that $\mathrm{ClHI}$ classified as being either chronic medical conditions, mental health conditions or cancer (Appendix 2, Table A2-2, contains details on the conditions and their classifications).

\section{Estimation of costs}

We estimated costs from the perspective of the public payer, the Ontario MOHLTC. Key components of what we denoted as "actual costs" in the prediction year were estimated according to commonly accepted costing methods ${ }^{12}$ and were aligned with those used by $\mathrm{CIHI}$. These values approximated those used by $\mathrm{CIHI}$ in model development and covered the same health care sectors (Appendix 3, available at www.cmaj.ca/lookup/suppl/ doi:10.1503/cmaj.191297/-/DC1). ${ }^{10}$

\section{Statistical analysis}

For analyses of model performance, we selected thresholds of actual cost representing high-cost users (top 10\%, top 5\% and top $1 \%$ ), because users at these thresholds have been found in prior studies to account for the majority of health care expenditures. ${ }^{1-7}$ Similarly, risk thresholds were defined for individuals in the top $10 \%, 5 \%$ and $1 \%$ in terms of predicted cost. At each threshold, we assessed the ability of the model to discriminate between higher and lower cost-risk individuals and examined how well the model was calibrated in terms of the difference between predicted and observed costs.

For model discrimination, we calculated the sensitivity (ability of the model to correctly select high-cost cases), specificity (ability of the model to accurately exclude low-cost individuals), positive predictive value (PPV; the proportion of cases predicted to be high cost that actually were high cost) and accuracy (correctly predicted cases - both high and lower cost - as a proportion of all individuals). We plotted the trade-off between sensitivity and specificity for each cost threshold using receiver operating characteristic curves. Strong model performance is indicated by values of the c-statistic, or area under the curve (AUC), of 0.8 or greater. ${ }^{13}$ We also calculated the Brier score, ${ }^{14}$ which measures the mean squared prediction error, where lower scores are better (within the range $0-1$ ). We conducted sensitivity analyses by varying the population as follows: including individuals with no health care utilization in the concurrent (baseline) period or excluding individuals who died during the prospective period (i.e., those whose baseline data were collected within 12 months before their death). 
Accuracy of the absolute estimates - or model calibration was assessed by plotting predicted versus actual cost and visually examining the divergence at different levels of actual cost.

\section{Ethics approval}

Formal ethics approval was not required because this study used de-identified administrative health care data that were obtained from the Ontario MOHLTC under an agreement with the Ontario Medical Association.

\section{Results}

The Registered Persons Database included 13293352 people aged younger than 106 years as of the end of the concurrent period (Mar. 31, 2016) who were residents of Ontario during fiscal years 2014/15 and 2015/16, who had at least 1 encounter with the health care system in the prior 5 years and who were eligible for OHIP coverage in fiscal year 2016/17. Limiting the analyses to those with health care encounters during the baseline period removed 847070 individuals (6.4\% of the initial population). Removing late entrants and early exits $(n=761855)$ to ensure sufficient data for prediction resulted in the exclusion of a further $5.7 \%$ of the initial population. The final study population consisted of 11684427 individuals who met all inclusion and exclusion criteria.

To highlight high-cost cases, we focused on the 90th, 95th and 99th percentiles of actual cost in the prospective period. Ten percent of the population ( $n=1168441)$ were estimated to have costs in excess of $\$ 3050$ per person in fiscal year 2016/17, accounting for $71.6 \%$ of total expenditures; $5 \%(n=584222)$ had costs greater than $\$ 6374$, accounting for $58.2 \%$ of total expenditures; and $1 \%(n=116845)$ had costs exceeding $\$ 22995$, accounting for $30.5 \%$ of total expenditures.

\section{Clinical profile of high-cost users}

Table 1 provides descriptive information about the individuals predicted to have high costs compared with those who actually had high costs in the prospective period. The prevalence of all

Table 1: Patient demographic characteristics and clinical complexity (2-year prevalence of chronic conditions) by risk score and prospective cost thresholds

\begin{tabular}{|c|c|c|c|c|c|c|c|c|}
\hline \multirow[b]{2}{*}{ Characteristic } & \multicolumn{4}{|c|}{ Risk score threshold* } & \multicolumn{4}{|c|}{ Cost threshold $\dagger$} \\
\hline & $\begin{array}{c}\geq 99 \text { th } \\
\text { percentile } \\
\text { (top } 1 \% \text { by } \\
\text { risk score) }\end{array}$ & $\begin{array}{l}\geq 90 \text { th } \\
\text { percentile } \\
\text { (top } 10 \% \text { by } \\
\text { risk score) }\end{array}$ & $\begin{array}{l}\geq 50 \text { th } \\
\text { percentile } \\
\text { (top } 50 \% \text { by } \\
\text { risk score) }\end{array}$ & $\begin{array}{c}<50 \text { th } \\
\text { percentile } \\
\text { (bottom } 50 \% \\
\text { by risk score) }\end{array}$ & $\begin{array}{c}\geq 99 \text { th } \\
\text { percentile } \\
\text { (top } 1 \% \\
\text { by cost) }\end{array}$ & $\begin{array}{c}\geq 90 \text { th } \\
\text { percentile } \\
\text { (top } 10 \% \\
\text { by cost) }\end{array}$ & $\begin{array}{c}\geq 50 \text { th } \\
\text { percentile } \\
\text { (top } 50 \% \\
\text { by cost) }\end{array}$ & $\begin{array}{c}<50 \text { th } \\
\text { percentile } \\
\text { (bottom } 50 \% \\
\text { by cost) }\end{array}$ \\
\hline \multicolumn{9}{|l|}{ Demographic } \\
\hline Age, yr, mean \pm SD & $71.0 \pm 16.7$ & $63.8 \pm 19.9$ & $51.6 \pm 21.6$ & $32.5 \pm 19.7$ & $66.8 \pm 18.4$ & $56.2 \pm 21.4$ & $49.0 \pm 22.4$ & $35.2 \pm 21.0$ \\
\hline Sex, female, \% & 47.3 & 55.2 & 55.4 & 49.4 & 46.4 & 58.1 & 57.0 & 47.8 \\
\hline \multicolumn{9}{|l|}{ Clinical complexity, \% } \\
\hline$\geq 1$ chronic conditions & 100.0 & 98.5 & 89.0 & 24.4 & 93.0 & 84.6 & 74.4 & 39.1 \\
\hline$\geq 2$ chronic conditions & 99.9 & 89.8 & 56.9 & 2.5 & 79.4 & 63.5 & 46.8 & 12.7 \\
\hline$\geq 3$ chronic conditions & 99.0 & 74.8 & 31.2 & 0.1 & 63.9 & 44.6 & 27.3 & 4.0 \\
\hline$\geq 5$ chronic conditions & 84.7 & 36.7 & 8.9 & 0.0 & 36.1 & 19.2 & 8.4 & 0.5 \\
\hline$\geq 10$ chronic conditions & 14.5 & 1.9 & 0.4 & 0.0 & 4.7 & 1.4 & 0.4 & 0.0 \\
\hline \multicolumn{9}{|l|}{ Severity, $\%$ with any } \\
\hline Minor chronic & 90.4 & 78.4 & 66.3 & 17.4 & 75.1 & 65.7 & 56.4 & 27.5 \\
\hline Moderate chronic & 77.5 & 52.6 & 28.3 & 1.7 & 49.0 & 36.7 & 23.6 & 6.4 \\
\hline Major chronic & 92.7 & 52.9 & 17.6 & 0.0 & 52.2 & 27.1 & 14.9 & 2.7 \\
\hline Major mental health & 27.7 & 17.8 & 5.0 & 0.0 & 12.6 & 7.6 & 4.3 & 0.8 \\
\hline Other mental health & 38.7 & 30.7 & 26.1 & 6.1 & 27.2 & 27.3 & 22.3 & 9.9 \\
\hline Cancer (any type) & 35.9 & 26.5 & 10.5 & 0.5 & 24.2 & 15.2 & 9.0 & 1.9 \\
\hline \multicolumn{9}{|c|}{ 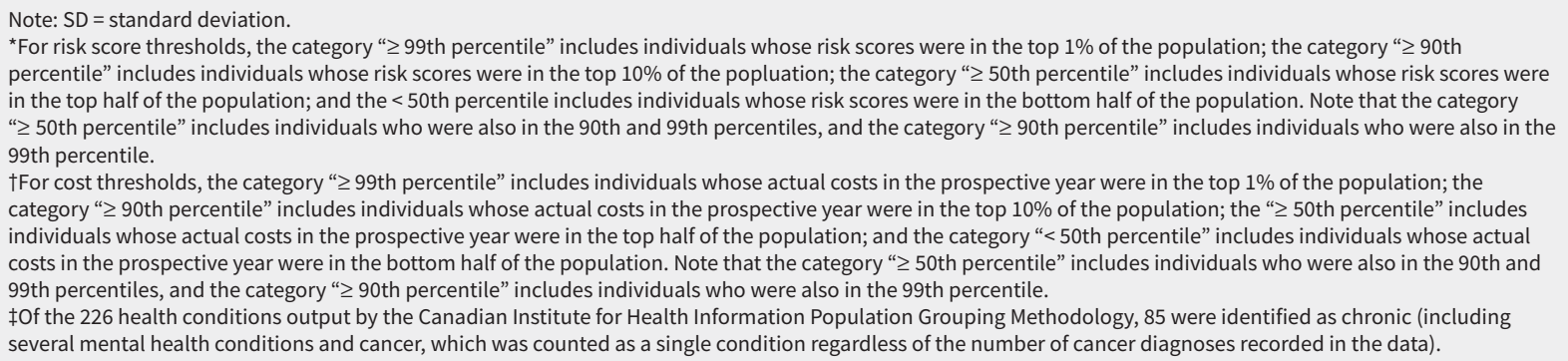 } \\
\hline
\end{tabular}


categories of chronic disease and multimorbidity (i.e., having 2 or more chronic conditions) rose starkly with predicted risk and actual cost. Just over one-tenth of those in the bottom half of the actual cost distribution had multiple comorbidities versus almost $80 \%$ of those in the top $1 \%$ cost group.

\section{Model performance}

Model discrimination measures for selected risk score cut points and cost thresholds are presented in Table 2. Model performance increased with higher cost thresholds, with AUC values of 0.78 (good performance), 0.81 (strong) and 0.86 (very strong) for the 90th, 95th and 99th percentile thresholds, respectively, indicating that cost thresholds became increasingly predictable in relation to prior diagnoses. Performance was somewhat affected by varying the population considered. The AUC value was slightly lower at the 95th percentile of cost when deaths were excluded from the analysis (AUC 0.79) compared with both the main analysis and the analysis that included non-users (AUC 0.81). Detailed results of the sensitivity analyses are presented in Appendix 4 (available at www.cmaj.ca/lookup/suppl/doi:10.1503/ cmaj.191297/-/DC1).

Model calibration is illustrated in Figure 1. An ideal model would follow the dashed $45^{\circ}$ line, whereby the observed

Table 2: CIHI Population Grouping Methodology model performance evaluated at the top $10 \%, 5 \%$ and $1 \%$ cost thresholds, by select risk score cut points

\section{Risk score percentile; criterion value}

\begin{tabular}{|c|c|c|c|}
\hline $\begin{array}{l}\text { Threshold of actual cost } \\
\text { in prospective period }\end{array}$ & 90th & 95th & 99th \\
\hline & \multicolumn{3}{|c|}{ Sensitivity* } \\
\hline Top $10 \%$ & 0.391 & 0.236 & 0.064 \\
\hline Top 5\% & 0.464 & 0.311 & 0.098 \\
\hline \multirow[t]{2}{*}{ Top 1\% } & 0.629 & 0.494 & 0.206 \\
\hline & \multicolumn{3}{|c|}{ Specificity† } \\
\hline Top 10\% & 0.928 & 0.968 & 0.996 \\
\hline Top 5\% & 0.919 & 0.964 & 0.995 \\
\hline \multirow[t]{2}{*}{ Top $1 \%$} & 0.905 & 0.954 & 0.992 \\
\hline & \multicolumn{3}{|c|}{ Positive predictive value $¥$} \\
\hline Top $10 \%$ & 0.385 & 0.463 & 0.631 \\
\hline Top 5\% & 0.232 & 0.311 & 0.491 \\
\hline \multirow[t]{2}{*}{ Top 1\% } & 0.063 & 0.099 & 0.206 \\
\hline & \multicolumn{3}{|c|}{ Accuracy§ } \\
\hline Top 10\% & 0.872 & 0.892 & 0.899 \\
\hline Top 5\% & 0.896 & 0.931 & 0.950 \\
\hline Top 1\% & 0.903 & 0.950 & 0.984 \\
\hline \multicolumn{4}{|c|}{$\begin{array}{l}\text { Note: } \mathrm{FN}=\text { false negatives, } \mathrm{FP}=\text { false positives, } \mathrm{TN}=\text { true negatives, } \mathrm{TP}=\text { true positive } \\
\text { *Sensitivity defined as proportion of high-cost cases predicted correctly (TP/[TP + FN } \\
\text { †Specificity defined as proportion of lower-cost cases predicted correctly (TN/[TN + FP] } \\
\text { †Positive predictive value defined as proportion of predicted high-cost cases that we } \\
\text { high cost (TP/[TP + FP]). } \\
\text { \$Accuracy defined as proportion of all cases predicted correctly, both high-cost and } \\
\text { lower-cost ([TP + TN]/[TP + FP + TN + FN]). }\end{array}$} \\
\hline
\end{tabular}

probability matches the predicted probability at every point. Because high-cost cases are rare events, most cases have low prediction probabilities (shown on the histograms in the bottom panel). The discrepancy between the logistic models and the ideal model increases with the prediction probability, yet the curves are all monotonically increasing, which means that the models are consistently predicting more high-cost cases with higher prediction probabilities. In each of the top $10 \%$, the top $5 \%$ and the top $1 \%$ scenarios, both Brier and AUC scores showed a moderate-to-strong fit of the model. Nevertheless, performance at the high end of the charts - which is most relevant for policy purposes - was relatively poor compared with performance at the low end.

\section{Interpretation}

Heavy users of health care services have received a great deal of attention recently, as governments are concerned about the large and rising share of public expenditures devoted to health care. We found that the top $10 \%$ of high-cost cases accounted for almost three-quarters of total expenditures, and the top $1 \%$ subset accounted for nearly one-third. The accuracy of the $\mathrm{CIHI}$ model improved with increasing cost threshold. We found that chronic, serious conditions were common among those in the top percentiles of risk and cost, along with increased rates of multimorbidity, indicating that part of the utilization among the highest-cost users was predictable.

The Ontario MOHLTC previously created a high-cost user model (for internal use only), which included demographic variables (age, sex, rural v. urban residence), clinical variables (primarily ICD-10 chapters), socioeconomic status variables and health care utilization variables for the current and prior 2 years. ${ }^{9}$ Performance of the $\mathrm{CIHI}$ model (without modifications) at the top $5 \%$ cost threshold, as reported here, was not quite as good as the MOHLTC's custom-built, high-cost case model. The c-statistic was 0.81 (strong), compared with 0.87 (very strong) for the MOHLTC model. Sensitivity at the top 5\% risk threshold was $31.1 \%$ (v. $42.2 \%$ ), specificity was $96.4 \%$ (v. $97.0 \%$ ), positive predictive value was $31.1 \%$ (v. $42.6 \%$ ), and model accuracy (true positives plus true negatives as a percentage of all predictions) was $93.1 \%$ (v. 94.2\%). A purposedesigned high-cost model built upon the $\mathrm{CIHI}$ diagnosis grouping methodology platform with the addition of other predictors of high cost utilization (e.g., prior cost) may be expected to boost performance to levels comparable with, or exceeding, those reported elsewhere. ${ }^{9,15}$

Although the purpose-designed MOHLTC model had superior performance, health risk predictive modelling tools like CIHI's Population Grouping Methodology have been used in other jurisdictions to select cases for targeted care initiatives. For example, the Vermont Chronic Care Initiative reported savings net of program expenses in excess of US\$30 million for fiscal year $2014 / 15,{ }^{16}$ which illustrates the potential value of such tools in supporting targeted programs to improve care coordination for residents eligible for state-funded services. ${ }^{15}$ It is noteworthy that PPV - perhaps the most important metric in this 
A
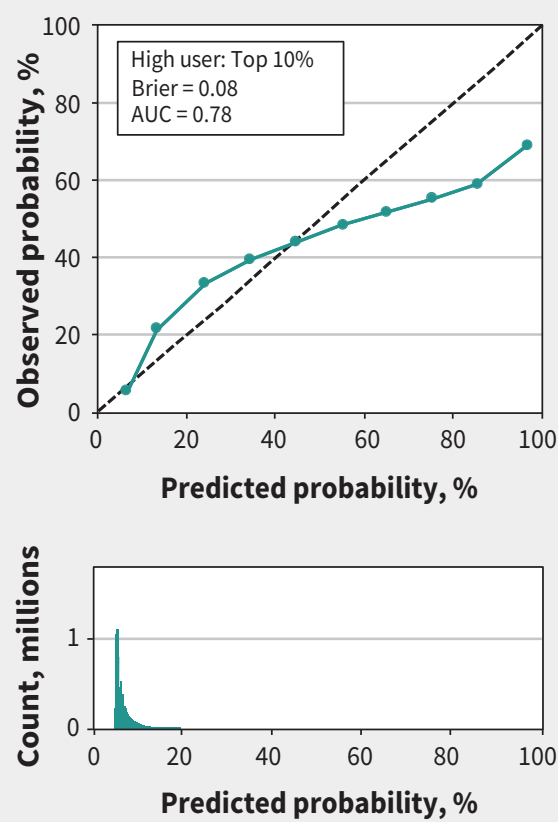

B
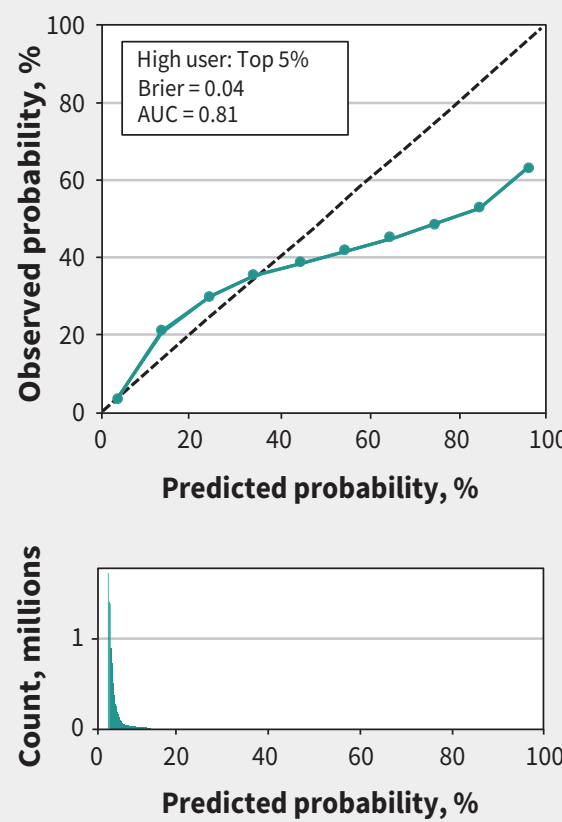

C
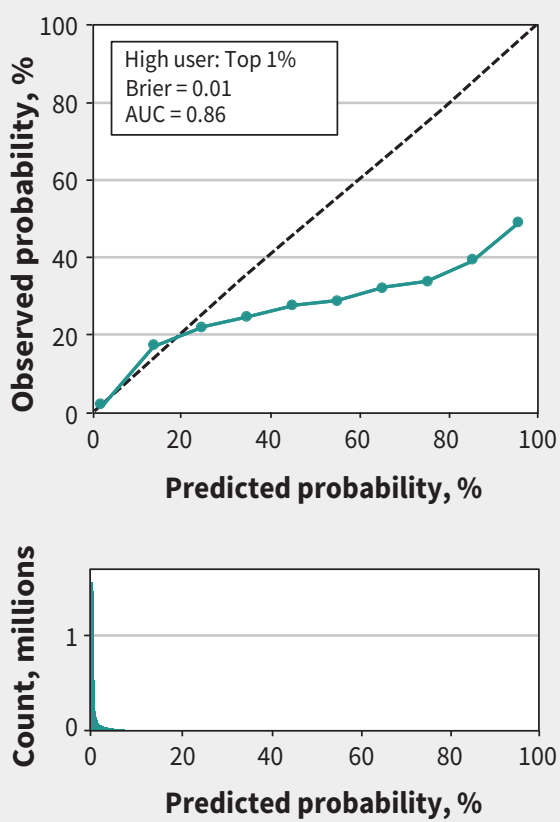

Figure 1: Model calibration, as predicted versus observed probabilities, for the top 10\% (A), top 5\% (B) and top 1\% (C) cost thresholds. The Brier score (ranging from 0 to 1 ) measures the mean squared prediction error, where lower scores are better. For area under the receiver operating characteristic curve (AUC), values of 0.8 or greater indicate strong model performance. The population is mostly concentrated in the lower range of predicted cost, where the model for the top $1 \%$ performs best; as such, although this model is less well calibrated at the top end, it does the best overall in terms of the Brier score and AUC.

context, given that it indicates the percentage of accurately predicted high-cost users - for the $\mathrm{CIHI}$ grouping methodology at the 90th percentile cost threshold and 95th percentile of risk (PPV 0.463) was much higher than that reported in a prior study evaluating the case selection tool that was used in Vermont in 2012 (PPV 0.35; relevant model, Chronic Illness and Disability Payment System). ${ }^{15}$ Like the conceptually equivalent $\mathrm{CIHI}$ tool, that model was not purpose-designed to predict the highestcost cases, yet it served the purposes of the Vermont Chronic Care Initiative effectively.

The CIHI Population Grouping Methodology performed adequately for predicting high-cost users and may provide a helpful initial filter to select cases for interventions to enhance health care delivery to those in high need and potentially to contain future health care expenditures. Although we recommend the development of a purpose-designed tool to improve model performance, the existing $\mathrm{CIHI}$ Population Grouping Methodology may be used - as is or in concert with additional information for many applications, such as case management and care coordination, that require prediction of future high-cost users.

\section{Limitations}

This study had several limitations. Ideally, costs would be estimated from a societal perspective and would include out-ofpocket expenditures by patients, formal and informal caregiving, and other relevant costs. However, data needed to include these costs were not available, both in the creation of the $\mathrm{CIHI}$ cost model and in our own analyses. In addition, neither the $\mathrm{CIHI}$ model nor our analyses included costs associated with inpatient mental health stays, inpatient rehabilitation, home care or longterm care.

Our estimates of total health care costs were lower than those reported in another recent study for Ontario, for which data were available from all health care settings. ${ }^{1}$ This was most apparent for the highest-cost users, who have been shown by prior research to be heavier-than-average users of non-acute hospital care, continuing care services and mental health care, ${ }^{1,17}$ the settings that were not available for our study. The top $1 \%$ of highcost users in the prior study had expenditures exceeding \$44906 in fiscal year 2011/12, which was more than double our result of $\$ 22254$ in fiscal year 2016/17, even without accounting for inflation. Nevertheless, the top $1 \%$ accounted for a similar percentage of expenditures (almost 31\% in our data v. 33\% in the prior study). Although CIHI's grouping methodology appears to be robust to incomplete data availability, ${ }^{11}$ data limitations may have partially obscured the clinical profiles of some of the highest-cost users. Model performance might have been better if complete diagnostic information from all settings had been available for this study.

\section{Conclusion}

Although the $\mathrm{ClHI}$ model was optimized to maximize predictive performance for the average user of health care services, on the basis of only past diagnoses of health conditions, the model performed only slightly worse than the purpose-designed model used by the MOHLTC for predicting high-cost users. CIHI may 
wish to consider inclusion of a purpose-designed model in future releases of its Population Grouping Methodology. Until then, the current model provides clinical condition information and risk scores that may provide a practical starting point for classification of patients according to cost risk.

\section{References}

1. Wodchis WP, Austin PC, Henry DA. A 3-year study of high-cost users of health care. CMAJ 2016;188:182-8.

2. Rais S, Nazerian A, Ardal S, et al. High-cost users of Ontario's healthcare services. Healthc Policy 2013;9:44-51.

3. Kozyrskyj A, Lix L, Dahl M, et al. High-cost users of pharmaceuticals: Who are they? Winnipeg: University of Manitoba, Faculty of Medicine, Department of Community Health Sciences, Manitoba Centre for Health Policy; 2005. Available: http://mchp-appserv.cpe.umanitoba.ca/reference/high-cost.pdf (accessed 2018 May 2).

4. Deber RB, Lam KCK. Handling the high spenders: implications of the distribution of health expenditures for financing health care. In: Chambers S, Jentleson BW, editors. Proceedings of the 2009 American Political Science Association annual meeting; 2009 Sept. 3-6; Toronto. Rochester (NY): Social Science Research Network; 2009. Available: https://papers.ssrn.com/sol3/papers.cfm?abstract_ id=1450788 (accessed 2018 May 2).

5. Roos NP, Shapiro E, Tate R. Does a small minority of elderly account for a majority of health care expenditures? A sixteen year perspective. Milbank $Q$ 1989;67:347-69.

6. Reid R, Evans R, Barer M, et al. Conspicuous consumption: characterizing high users of physician services in one Canadian province. J Health Serv Res Policy 2003;8: 215-24.
7. Briggs T, Burd M, Fransoo R. Identifying high users of healthcare in British Columbia, Alberta and Manitoba. Healthc Pap 2014;14:31-6, discussion 58-60.

8. Conway P, Goodrich K, Machlin S, et al. Patient-centered care categorization of U.S. health care expenditures. Health Serv Res 2011;46:479-90.

9. Chechulin $\mathrm{Y}$, Nazerian A, Rais S, et al. Predicting patients with high risk of becoming high-cost healthcare users in Ontario (Canada). Healthc Policy 2014;9:68-79.

10. Population grouping methodology [information sheet]. Ottawa: Canadian Institute for Health Information; 2017.

11. Li Y, Weir S, Steffler M, et al. Using diagnoses to estimate health care cost risk in Canada. Med Care 2019;57:875-81.

12. Wodchis WP, Bushmeneva K, Nikitovic M, et al. 4.8. Physician services. In: Guidelines on person-level costing using administrative databases in Ontario. Working Paper Series Vol. 1:35-6. Toronto: Health System Performance Research Network; 2013. Available: https://tspace.library.utoronto.ca/ bitstream/1807/87373/1/Wodchis\%20et\%20al_2013_Guidelines\%20on\%20 Person-Level\%20Costing.pdf (accessed 2020 Apr. 2).

13. Hosmer DW, Lemeshow S. Applied logistic regression. 2nd ed. New York: John Wiley \& Sons; 2000.

14. Rufibach K. Use of Brier score to assess binary predictions [letter]. J Clin Epidemiol 2010;63:938-9.

15. Weir S, Aweh G, Clark RE. Case selection for a Medicaid chronic care management program. Health Care Financ Rev 2008;30:61-74.

16. 7.6: Vermont Chronic Care Initiative (VCCI). In: Vermont blueprint for health 2015 annual report. Waterbury (VT): Government of Vermont; 2016. p. 64-7. Available: https://ljo.vermont.gov/assets/docs/healthcare/Health-Reform -Oversight-Committee/2016-Interim-Reports/da34dd8eeb/2015-Annual-Blue print-Report.pdf (accessed 2020 Apr. 2).

17. Anderson M, Revie CW, Quail JM, et al. The effect of socio-demographic factors on mental health and addiction high-cost use: a retrospective, populationbased study in Saskatchewan. Can J Public Health 2018;109:810-20.

\section{Competing interests: None declared.}

This article has been peer reviewed.

Affiliation: Economics, Policy and Research, Ontario Medical Association, Toronto, Ont.

Contributors: Sharada Weir conceived the study and developed the analysis plan. Jasmin Kantarevic acquired the data. Sharada Weir, Mitch Steffler and Yin Li analyzed the data. Sharada Weir drafted the manuscript. All of the authors contributed to interpretation of the data, revised the manuscript for important intellectual content, approved the final version for publication and agreed to act as guarantors of the work.

\section{Funding: None received.}

Data sharing: The data set from this study is held securely at the Ontario Medical Association (OMA) under a data-sharing agreement with the Ontario Ministry of Health and LongTerm Care that prohibits the OMA from making the data set publicly available.

Acknowledgements: The Population Grouping Methodology is owned by the Canadian Institute for Health Information (CIHI) and was used under licence. Population-level health care data were obtained from the Ontario Ministry of Health and Long-Term Care (MOHLTC) under an agreement with the Ontario Medical
Association. Both $\mathrm{CIHI}$ and the MOHLTC had no involvement in or control over the design and conduct of the study; the collection, analysis and interpretation of the data; the preparation of the data; the decision to publish; or the preparation, review and approval of the manuscript. The views expressed in this paper are strictly those of the authors. No official endorsement by the Ontario Medical Association is intended or should be inferred.

Accepted: Mar. 27, 2020

Correspondence to: Sharada Weir, sharada. weir@oma.org 